\title{
Structural Sparse Tracking
}

\author{
Tianzhu Zhang ${ }^{1,2} \quad \mathrm{Si} \mathrm{Liu}^{3} \quad$ Changsheng $\mathrm{Xu}^{2,4}$ Shuicheng Yan $^{5} \quad$ Bernard Ghanem $^{1,6}$ \\ Narendra Ahuja ${ }^{1,7}$ Ming-Hsuan Yang ${ }^{8}$ \\ ${ }^{1}$ Advanced Digital Sciences Center ${ }^{2}$ Institute of Automation, CAS ${ }^{3}$ Institute of Information Engineering, CAS \\ ${ }^{4}$ China-Singapore Institute of Digital Media ${ }^{5}$ National University of Singapore ${ }^{6}$ King Abdullah University of Science and Technology \\ ${ }^{7}$ University of Illinois at Urbana-Champaign ${ }^{8}$ University of California at Merced
}

\begin{abstract}
Sparse representation has been applied to visual tracking by finding the best target candidate with minimal reconstruction error by use of target templates. However, most sparse representation based trackers only consider holistic or local representations and do not make full use of the intrinsic structure among and inside target candidates, thereby making the representation less effective when similar objects appear or under occlusion. In this paper, we propose a novel Structural Sparse Tracking (SST) algorithm, which not only exploits the intrinsic relationship among target candidates and their local patches to learn their sparse representations jointly, but also preserves the spatial layout structure among the local patches inside each target candidate. We show that our SST algorithm accommodates most existing sparse trackers with the respective merits. Both qualitative and quantitative evaluations on challenging benchmark image sequences demonstrate that the proposed SST algorithm performs favorably against several state-of-the-art methods.
\end{abstract}

\section{Introduction}

Visual tracking aims to estimate the states of a moving target in a video. It has long been one of the most important and fundamental topics in computer vision with a plethora of applications such as surveillance, vehicle navigation, human computer interface, and human motion analysis, to name a few. Despite numerous object tracking methods [33, 26, 35, 30, 17, 24] having been proposed in recent years, it remains a challenging task to develop a robust algorithm for complex and dynamic scenes due to the factors such as partial occlusions, illumination, pose, scale, camera motion, background clutter, and viewpoint.

Most tracking algorithms are developed from the discriminative or generative perspectives. Discriminative approaches formulate the tracking problem as a binary classification task in order to find the decision boundary for separating the target object from the background [2, 6, 9, 10, 3, 16]. Avidan [2] combines a set of weak classifiers into a strong one and develops an ensemble tracking method. Collins et al. [6] demonstrate that the most discriminative features can be learned online to separate the target object from the background. In [9], Grabner et al. propose an online boosting method to update discriminative features and a semi-online boosting algorithm [10] to handle the drifting problem in object tracking. Babenko et al. [3] introduce multiple instance learning into online object tracking where samples are considered within positive and negative bags or sets. Kalal et al. [16] propose the P-N learning algorithm to exploit the underlying structure of positive and negative samples to learn classifiers for object tracking.

In contrast, generative tracking methods typically learn a model to represent the target object and then use it to search for the image region with minimal reconstruction error [5, 7, 14, 1, 25, 31, 18]. Black et al. [5] learn an off-line subspace model to represent the object of interest for tracking. The mean shift tracking algorithm [7] models a target with nonparametric distributions of features (e.g., color pixels) and locates the object with mode shifts. The Frag tracker [1] addresses the partial occlusion problem by modeling object appearance with histograms of local patches. The IVT method [25] utilizes an incremental subspace model to adapt appearance changes. Kwon et al. [18] use multiple observation models to cover a wide range of appearance changes, caused by pose and illumination variation, for tracking. Most of these methods use holistic representations to describe objects and hence do not handle occlusions or distracters well.

Recently, sparse representation based generative tracking methods have been developed for object tracking [21, 20, 19, 23, 22, 34, 37, 36, 4, 15, 13, 39, 42, 41, 29]. These trackers can be categorized based on the representation schemes into global, local, and joint sparse appearance models as shown in Figure 1. In [21, 19, 23, 22, 34, 4], the sparse trackers represent each target candidate $\mathbf{x}_{i}$ as a sparse linear combination of target templates $\mathbf{T}$ that can be dynamically updated to account for appearance changes. These models have been shown to be robust against partial occlusions with demonstrated performance for tracking. However, all these methods model a target object as a single entity as shown in 


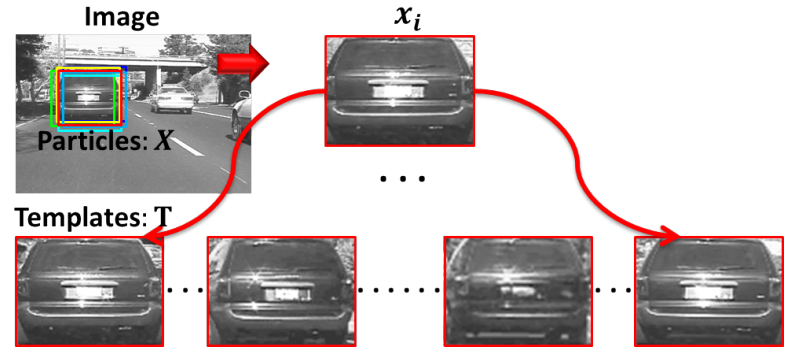

(a) global sparse appearance model

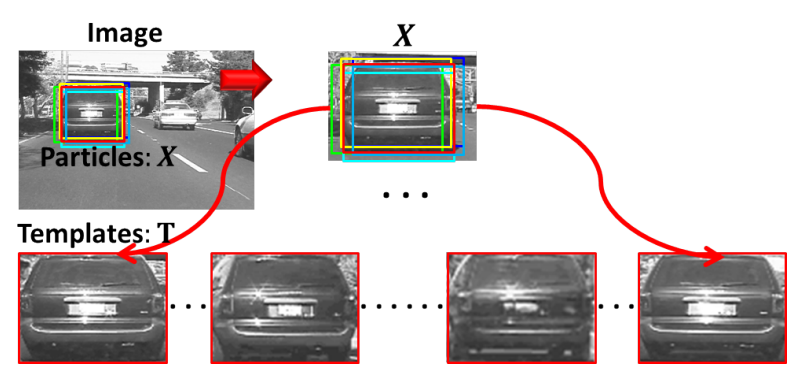

(c) joint sparse appearance model

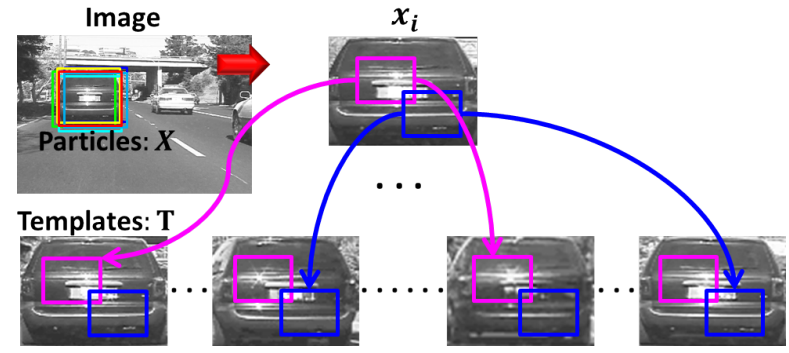

(b) local sparse appearance model

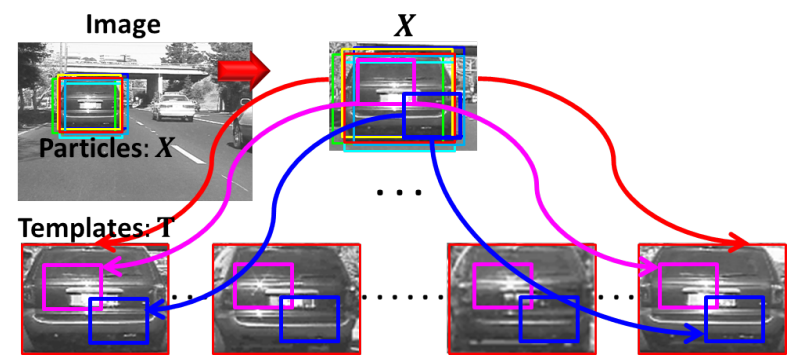

(d) structural sparse appearance model

Figure 1. Sparse representation based trackers [21, 20, 19, 23, 22, 34, 37, 36, 15, 13]. These methods are grouped based on their sparse appearance models. Given an image with the $n$ sampled particles $\mathbf{X}=\left[\mathbf{x}_{1}, \cdots, \mathbf{x}_{i}, \cdots, \mathbf{x}_{n}\right]$ and the dictionary templates $\mathbf{T}$. (a) Global sparse appearance model $[21,19,23,22,34]$. These trackers adopt the holistic representation of a target as the appearance model and tracking is carried out by solving $\ell_{1}$ minimization problems. As a result, the target candidate $\mathbf{x}_{i}$ is represented by a sparse number of elements in $\mathbf{T}$. (b) Local sparse appearance model [20,15]. These trackers represent each local patch inside one possible target candidate $\mathbf{x}_{i}$ by a sparse linear combination of the local patches in $\mathbf{T}$. Note that, the local patches inside the target candidate $\mathbf{x}_{i}$ may be sparsely represented by the corresponding local patches inside different dictionary templates. (c) Joint sparse appearance model [37, 36, 13]. These trackers exploit the intrinsic relationship among particles $\mathbf{X}$ to learn their sparse representations jointly. The joint sparsity constraints encourage all particle representations to be jointly sparse and share the same (few) dictionary templates that reliably represent them. (d) The proposed structural sparse appearance model incorporates the above three models together. Our model exploits the intrinsic relationship among particles $\mathbf{X}$ and their local patches to learn their sparse representations jointly. In addition, our method also preserves the spatial layout structure among the local patches inside each target candidate, which is ignored by the above three models [21, 19, 23, 22, 34, 20, 15, 37, 36, 4, 13]. Using our model, all particles $\mathbf{X}$ and their local patches are represented with joint sparsity, i.e., only a few (but the same) dictionary templates are used to represent all the particles and their local patches at each frame. Note that, the local patches inside all particles $\mathbf{X}$ are represented with joint sparsity by the corresponding local patches inside the same dictionary templates used to represent $\mathbf{X}$.

Figure 1(a), and adopt the global sparse appearance model. Therefore, these methods are less effective in handling heavy occlusions.

The basic idea of local sparse appearance models [20, 15] is shown in Figure 1(b) where local patches inside a possible target candidate $\mathbf{x}_{i}$ are sparsely represented with local patches in the dictionary templates. In [20], Liu et al. propose a tracking algorithm based on a local sparse model which employs histograms of sparse coefficients and the mean-shift algorithm for object tracking. However, this method is based on a static local sparse dictionary and likely to fail when similar objects appear in the scenes. Jia et al. [15] develop a tracking method based on a local sparse appearance model using a set of overlapped image patches inside the target region with a spatial layout. These local patches are used to form a dictionary for encoding regions inside the possible candidate regions. For a target candidate, its local patches are extracted in the same way. Since each local patch rep- resents one fixed part of the target object, the local patches altogether can represent the complete structure of the target. With the sparsity assumption, the local patches within the target candidate can be represented as the linear combination of only a few dictionary bases by solving an $\ell_{1}$ minimization problem. Although this model addresses some issues of global sparse appearance models, such tracking algorithm$\mathrm{s}[20,15]$ do not consider the spatial layout structure among the local patches inside each target candidate or the correlations among the local patches from all target candidates. For example, as shown in Figure 1(b), the local patches inside a target candidate $\mathbf{x}_{i}$ may be sparsely represented by the local patches from different dictionary templates. Ideally, to maintain the spatial layout among the local patches, the purple local patch of $\mathbf{x}_{i}$ is best represented by the local patch of the first dictionary basis, and the blue local patch of $\mathbf{x}_{i}$ should also be represented with the corresponding blue local patch of the first dictionary basis. 
The joint sparse appearance model $[37,36,13]$ is shown in Figure 1(c), which is motivated by the following observations. In particle filter-based tracking methods, particles at and around the target are randomly sampled according to a zero-mean Gaussian distribution based on the previous states. Each particle shares dependencies with other particles and their corresponding images are likely to be similar. In [37], learning the representation of each particle is viewed as an individual task and a multi-task learning with joint sparsity for all particles is employed. In [36], the low-rank sparse learning is applied to learn the sparse representations of all particles jointly. In [13], the multi-task multi-view join$\mathrm{t}$ sparse representation is adopted for tracking. The methods based on joint sparse appearance models [37, 36, 13, 38, 40] aim to improve the tracking performance by exploiting the intrinsic relationship among particles. Moreover, due to the joint optimization among all particles $\mathbf{X}$, this model is computationally efficient. However, such models still use the holistic representations to describe object appearance.

Motivated by the above three models, we propose a novel structural sparse appearance model as shown in Figure 1(d), which has the following differences. First, the proposed structural sparse appearance model incorporates the above three models together and is shown to be less sensitive to partial occlusion [20,15], and computationally efficien$\mathrm{t}[37,36,13]$ by considering the correlations among the target candidates. Second, the proposed model exploits the intrinsic relationship among not only the particles $\mathbf{X}$, but also the corresponding local image patches to learn their sparse representations jointly. Third, the proposed model preserves the spatial layout structure among the local patches inside each target candidate, which is ignored in the previous sparse trackers [21, 19, 23, 22, 34, 20, 15, 37, 36, 4, 13]. As shown in Figure 1(d), since all particles $\mathbf{X}$ and their local patches are represented with joint sparsity, only a few (but the same) dictionary templates are used to represent all the particles and their local patches at each frame. Note that, the local patches inside all particles $\mathbf{X}$ are represented with joint sparsity by the corresponding local patches inside the same dictionary templates used to represent $\mathbf{X}$.

Based on the structural sparse appearance model, we propose a computationally efficient structural sparse tracking (SST) algorithm within the particle filter framework. Here, all particles and their local patches are represented via the proposed structural sparse appearance model, and the next target state is the particle that it and its local patches have the highest similarity with target dictionary templates and their corresponding patches. Unlike previous methods, our proposed SST algorithm not only exploits the intrinsic relationship among particles and their local patches to learn their sparse representations jointly, but also preserves the spatial layout structure among the local patches inside each target candidate. This helps locate the target more accurately and is less sensitive to partial occlusion. In our SST formulation, we use the $\ell_{p, q}$ mixed-norm regularizer, which is optimized
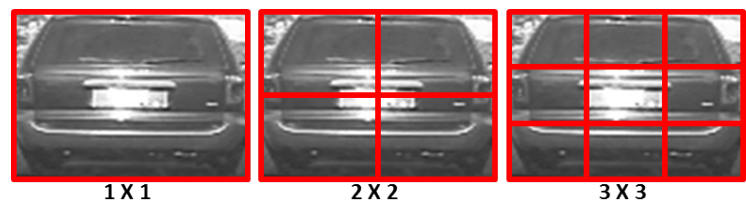

Figure 2. The spatial layout for local patches sampling. Note that, any other local patch sampling methods can also be adopted.

using an Accelerated Proximal Gradient (APG) method for fast convergence. In addition, we show that existing $\ell_{1}$ tracker [22], LST [15], and MTT [37] methods are the special cases of our SST formulation.

\section{Structural Sparse Tracking}

In this section, we give a detailed description of our particle filter based tracking method that use the structural sparse appearance model to represent particles and their local patches jointly. Particles are sampled at and around the previous object location to predict the state $\mathbf{s}_{t}$ of the target at time $t$, from which we crop the region of interest $\mathbf{y}_{t}$ in the current image and normalize it to the template size. The state transition function $p\left(\mathbf{s}_{t} \mid \mathbf{s}_{t-1}\right)$ is modeled by an affine motion model with a diagonal Gaussian distribution. The observation model $p\left(\mathbf{y}_{t} \mid \mathbf{s}_{t}\right)$ reflects the similarity between an observed image region $\mathbf{y}_{t}$ corresponding to a particle $\mathbf{s}_{t}$ and the templates of the current dictionary. In this work, $p\left(\mathbf{y}_{t} \mid \mathbf{s}_{t}\right)$ is computed by a function of the reconstruction error obtained by linearly representing $\mathbf{y}_{t}$ and its local patches using the template dictionary. The particle that maximizes this function is selected to be the tracked target at each time instance. Next, we show how to use the structural sparse appearance model to represent particles and their local patches in details.

\subsection{Structural Sparse Appearance Model}

Given the image set of the target templates $\mathbf{T}=$ $\left[\mathbf{T}_{1}, \mathbf{T}_{2}, \cdots, \mathbf{T}_{m}\right]$, we sample $K$ local image patches inside each target region with a spatial layout. For simplicity, the spatial layout as shown in Figure 2 is used. Note that, any other local patch sampling methods can also be adopted. After sampling local patches, they are used to form a dictionary for encoding local patches inside any candidate region. For the $k$-th local image patch among these $m$ target templates, we obtain the corresponding dictionary templates $\mathbf{D}^{k}=\left[\mathbf{d}_{1}^{k}, \mathbf{d}_{2}^{k}, \cdots, \mathbf{d}_{m}^{k}\right] \in \mathbb{R}^{d_{k} \times m}$, where $k=1, \cdots, K ;$ $K$ is the number of local patches sampled within the target region; $d_{k}$ is the dimension of the $k$-th image patch vector; and $m$ is the number of target templates. Each column in $\mathbf{D}^{k}$ is obtained by $\ell_{2}$ normalization on the vectorized grayscale image observations extracted from $\mathbf{T}$. Each local patch represents one fixed part of the target, and hence the local patches altogether can represent the complete structure of the target. Since the image patches are collected from many templates, this dictionary captures the commonality of different templates and is able to represent various forms of these part- 


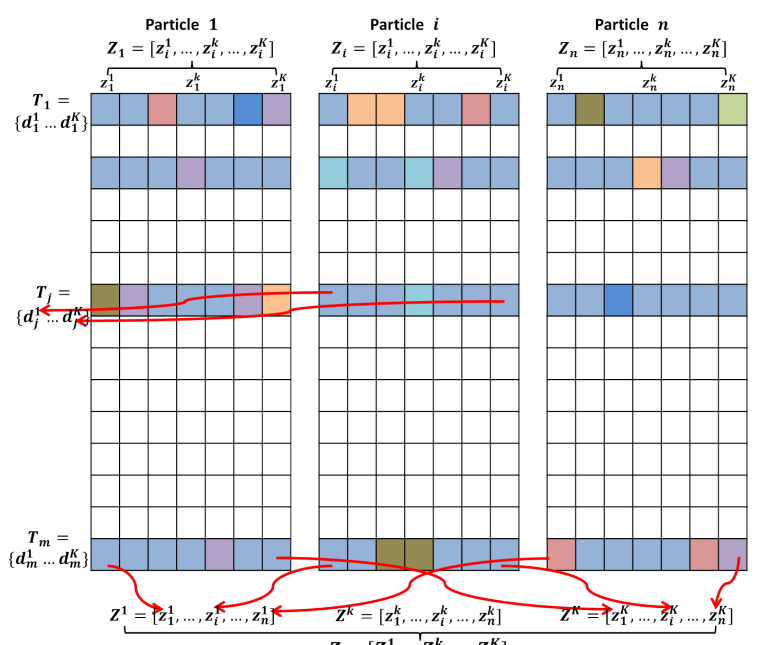

Figure 3. Illustration for the structure of the learned coefficient matrix $\mathbf{Z}$, where entries of different color represent different learned values, and the white entries indicate the zero rows and columns.

s. To incorporate variations in object appearance for robust tracking, the dictionary templates $\mathbf{T}$ are progressively updated. The dictionary update scheme in this work is similar to [22]. Each target template in $\mathbf{T}$ is assigned a weight that indicates how representative the template is. When a template is frequently used to represent tracking results, it has higher weight. When the set $\mathbf{T}$ does not represent particles well, the target template with the lowest weight is replaced by the current tracking result. To initialize the $m$ target templates, we sample equal-sized patches at and around the initial position of the target.

At time $t$, we have $n$ particle samples and the corresponding vectorized gray-scale image observations form a matrix $\mathbf{X}=\left[\mathbf{x}_{1}, \mathbf{x}_{2}, \cdots, \mathbf{x}_{n}\right]$, where the observation with respec$\mathrm{t}$ to the $i$-th particle is denoted as $\mathbf{x}_{i}$. For a target candidate $\mathbf{x}_{i}$, we extract $K$ local patches within it and convert them into vectors in the same way as how to construct dictionary templates $\mathbf{D}^{k}$. For the $k$-th local image patches of these $n$ particle samples, their corresponding vectorized gray-scale image observations form a matrix $\mathbf{X}^{k}=$ $\left[\mathbf{x}_{1}^{k}, \mathbf{x}_{2}^{k}, \cdots, \mathbf{x}_{n}^{k}\right] \in \mathbb{R}^{d_{k} \times n}$. We represent each observation from $\mathbf{X}^{k}$ by a linear combination of templates from the dictionary $\mathbf{D}^{k}$, such that $\mathbf{X}^{k}=\mathbf{D}^{k} \mathbf{Z}^{k}$. Here, the columns of $\mathbf{Z}^{k}=\left[\mathbf{z}_{1}^{k}, \mathbf{z}_{2}^{k}, \cdots, \mathbf{z}_{n}^{k}\right] \in \mathbb{R}^{m \times n}$ denote the representation$\mathrm{s}$ of the $k$-th local patch observations with respect to $\mathbf{D}^{k}$. Putting the representations of all the $K$ local patches together, we obtain $\mathbf{Z}=\left[\mathbf{Z}^{1}, \mathbf{Z}^{2}, \cdots, \mathbf{Z}^{K}\right] \in \mathbb{R}^{m \times n K}$. For the $i$-th particle, the corresponding representations of its local patches form a matrix $\mathbf{Z}_{i}=\left[\mathbf{z}_{i}^{1}, \mathbf{z}_{i}^{2}, \cdots, \mathbf{z}_{i}^{K}\right] \in \mathbb{R}^{m \times K}$.

For the $\mathbf{Z}^{k}$ and $\mathbf{Z}_{i}$, we have the following observations as shown in Figure 3: (1). For the $\mathbf{Z}^{k}$, it describes the representations of all the $k$-th image patches of the $n$ sampled particles. Based on the fact that these particles are densely sampled at and around the target, these particles are likely to be similar, and their $k$-th image patches are also expected
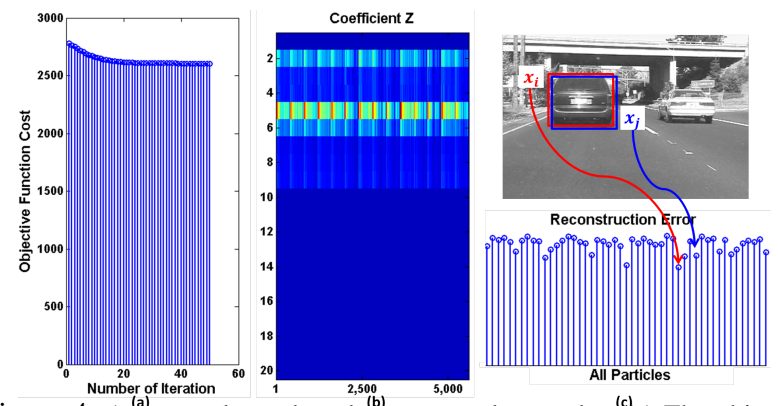

Figure 4. An example to show how our tracker works. (a) (a) The objective function cost vs the number of iteration. Our algorithm can converge after several iterations. (b) The learned matrix $\mathbf{X} \in \mathbb{R}^{20 \times 5600}$. Here, $m=20$, $K=14$, and $n=400$. Notice that the columns of $\mathbf{Z}$ are jointly sparse, i.e., a few (but the same) dictionary templates are used to represent all image patches together. (c) The particle $\mathbf{x}_{i}$ is selected among all other particles as the tracking result, since it has the smallest reconstruction error.

to be similar. Therefore, the underlying relationships among local patches should be exploited whereas existing methods based on local sparse representations [20,15] do not take these properties into account. (2). For the $\mathbf{Z}_{i}$, it shows the corresponding representations of all local patches of the $i$ th particle. Because these local patches are sampled inside the target candidate, their spatial layout structure should be preserved. Namely, after sampling these local patches via the spatial layout as shown in Figure 2, their representations should meet the following constraint: if the $k$-th image patch inside the $i$-th particle selects the $k$-th element of the target template $\mathbf{T}_{j}=\left\{\mathbf{d}_{j}^{1}, \mathbf{d}_{j}^{2}, \cdots, \mathbf{d}_{j}^{K}\right\}$ for representing $\mathbf{z}_{i}^{k}$, the other image patches should also select the corresponding elements in the same target template $\mathbf{T}_{j}$ for representations.

Motivated by the above observations, we use the convex $\ell_{p, q}$ mixed norm, especially, $\ell_{2,1}$ to model the structure information of $\mathbf{Z}^{k}$ and $\mathbf{Z}_{i}$ and obtain the structural sparse appearance model for object tracking as

$$
\min _{\mathbf{Z}} \frac{1}{2} \sum_{k=1}^{K}\left\|\mathbf{X}^{k}-\mathbf{D}^{k} \mathbf{Z}^{k}\right\|_{F}^{2}+\lambda\|\mathbf{Z}\|_{2,1}
$$

where $\mathbf{Z}=\left[\mathbf{Z}^{1}, \mathbf{Z}^{2}, \cdots, \mathbf{Z}^{K}\right] \in \mathbb{R}^{m \times n K},\|\cdot\|_{F}$ denotes the Frobenius norm, and $\lambda$ is a tradeoff parameter between reliable reconstruction and joint sparsity regularization. The definition of the $\ell_{p, q}$ mixed norm is $\|\mathbf{Z}\|_{p, q}=$ $\left(\sum_{i}\left(\sum_{j}\left|[\mathbf{Z}]_{i j}\right|^{p}\right)^{\frac{q}{p}}\right)^{\frac{1}{q}}$ and $[\mathbf{Z}]_{i j}$ denotes the entry at the $i$-th row and $j$-th column of $\mathbf{Z}$.

Figure 3 illustrates the structure of the learned matrix $\mathbf{Z}$. After learning the $\mathbf{Z}$, the observation likelihood of the tracking candidate $i$ is defined as $p\left(\mathbf{y}_{t} \mid \mathbf{s}_{t}\right)=$ $\frac{1}{\beta} \exp \left(-\alpha \sum_{k=1}^{K}\left\|\mathbf{x}_{i}^{k}-\mathbf{D}^{k} \mathbf{z}_{i}^{k}\right\|_{F}^{2}\right)$, where $\mathbf{z}_{i}^{k}$ is the coefficient of the $i$-th candidate corresponding to the target templates of the $k$-th image patch, and $\alpha$ and $\beta$ are normalization parameters. The tracking result is the particle that has the maximum observation likelihood.

For a more intuitive view of the proposed formulation, 
we visualize an empirical example of the learned sparse representation in Figure 4 and show an example of how the proposed SST tracker works. Given all particles X (sampled around the tracked car), their local patches $\left(\mathbf{X}^{k}, k=\right.$ $1, \cdots, K)$ can be sampled based on the spatial layout as shown in Figure 2. Then, based on the corresponding dictionary templates $\left(\mathbf{D}^{k}, k=1, \cdots, K\right)$, we can learn the representation matrix $\mathbf{Z}=\left[\mathbf{Z}^{1}, \mathbf{Z}^{2}, \cdots, \mathbf{Z}^{K}\right]$ by solving (1). Note that a brighter color squares in $\mathbf{Z}$ represents a larger value in the corresponding entry. Clearly, columns of $\mathbf{Z}$ are jointly sparse, i.e., a few (but the same) dictionary templates are used to represent all the image patches together. The particle $\mathbf{x}_{i}$ is determined as the current tracking result $\mathbf{y}_{t}$ because the reconstruction error of its image patches with respect to the target templates is the smallest among all particles. Since particle $\mathbf{x}_{j}$ corresponds to a misaligned image of the target, it has a bigger reconstruction error and cannot be represented well by $\mathbf{T}$.

\subsection{Discussion}

As discussed in the introduction and in Figure 1, existing sparse tracking methods $[21,20,19,23,22,34,37,36$, $15,13]$ can be categorized as global, local, and joint sparse appearance models based on the representation schemes. Motivated by the above three models, we propose a novel structural sparse appearance model as shown in (1) for object tracking. Our formulation (1) is generic and incorporates the above three models together to have their properties. It is worth emphasizing the difference between the proposed SST algorithm and several related tracking methods [21, 20, 19, 23, 22, 34, 37, 36, 15, 13].

- Global sparse appearance models for tracking [21, 19, 23, 22, 34]. In (1), when $K=1$ (only $1 \times 1$ as shown in Figure 2), with $\ell_{1,1}$ mixed norm is adopted, the formulation (1) reduces to a global sparse appearance model, which modes a target by one single entity, and learns the sparse representations of target candidates independently without considering their intrinsic relationships.

- Local sparse appearance models for tracking [20, 15]. In (1), with the image patch sampling methods as [20, 15] and the $\ell_{1,1}$ mixed norm, our formulation (1) reduces to a local sparse representation model, which does not consider the correlations of image patches among multiple target candidates or the spatial layout structure of image patches inside each target candidate.

- Joint sparse appearance model for tracking [37, 36, 13]. In (1), when $K=1$ (only $1 \times 1$ as shown in Figure 2), with $\ell_{2,1}$ mixed norm, the formulation (1) reduces to a joint sparse representation model, which considers the intrinsic relationships among target candidates. However, this model uses a holistic object representation.

- The structural sparse appearance model for tracking. Our tracker SST has the following properties: (1). It considers both the global and local sparsity constraints. (2). It considers the intrinsic relationships among not only the target candidates, but also their local image patches. (3). It considers the spatial layout structure of image patches inside each target candidate.

Based on the above discussion, it is clear that our formulation in (1) is generic and most tracking methods based on sparse representation are its special cases. Our formulation (1) does not only maintain the sparse properties of the existing three models, but also exploit the spatial layout structure of image patches inside each target candidate.

\section{Experimental Results}

To evaluate the performance of our tracker, we conduct extensively experiments on 20 publicly available challenging image sequences. These sequences contain complex scenes with challenging factors for visual tracking, e.g., cluttered background, moving camera, fast movement, large variation in pose and scale, partial occlusion, shape deformation and distortion (See Figure 5). For comparison, we run 14 stateof-the-art algorithms with the same initial position of the target. These algorithms are the online multiple instance learning (MIL) [3], online Adabost boosting (OAB) [9], tracking by detection (TLD) [16], Struck [11], circulant structure tracking (CST) [12], part-based visual tracking (PT) [32], real time compressive tracking (RTCT) [34], $\ell_{1}$ tracking $\left(\ell_{1} \mathrm{~T}\right)$ [22], local sparse tracking (LST) [15], multi-task tracking (MTT) [37], incremental visual tracking (IVT) [25], distribution field tracking (DFT) [27], fragments-based (Frag) [1], and local-global tracking (LGT) [28] methods. Here, MIL, OAB, TLD, Struck, CST, and PT are discriminative trackers, and others (IVT, DFT, Frag, LGT, RTCT, $\ell_{1}$ T, MTT, and LST) are generative trackers. In addition, RTCT and $\ell_{1}$ T, LST, and MTT are based on global, local, and joint sparse models, respectively. By comparing with these different kinds of methods, we demonstrate the effectiveness of our proposed SST. For fair comparisons, we use the publicly available source or binary codes provided by the authors. The default parameters are set for initialization.

For all reported experiments, we set $\eta=0.1, \widetilde{\lambda}=0.5$, the number of image patches $K=14$ as shown in Figure 2, the number of templates $m=20$, the number of particles $n=400$ (the same for $\ell_{1} \mathrm{~T}$ and MTT). The variances of affine parameters for particle sampling is set to $(0.01,0.0005,0.0005,0.01,4,4)$. The template size $d$ is set to half the size of the target object manually initialized in the first frame. The proposed algorithm is implemented in MAT$\mathrm{LAB}$ and runs at 0.45 seconds per frame on a $2.80 \mathrm{GHz}$ Intel Core2 Duo machine with 8GB memory. We will make the source code available to the public.

\subsection{Quantitative Evaluation}

To quantitatively evaluate the performance of each tracker, we use two metrics including the center location error 
Table 1. The average overlap score of 15 different trackers on 20 different videos. On average, the proposed tracker SST outperforms the other 14 state-of-the-art trackers. For each video, the smallest and second smallest distances are denoted in red and blue, respectively.

\begin{tabular}{|c|c|c|c|c|c|c|c|c|c|c|c|c|c|c|c|}
\hline Video & SST & RTCT & IVT & MIL & OAB & Frag & Struck & MTT & $\ell_{1} \mathrm{~T}$ & TLD & CST & DFT & LST & PT & LGT \\
\hline tunnel & 0.64 & 0.29 & 0.21 & 0.08 & 0.09 & 0.04 & 0.32 & 0.23 & 0.15 & 0.34 & 0.32 & 0.23 & 0.63 & 0.31 & 0.15 \\
\hline tud & 0.87 & 0.32 & 0.56 & 0.38 & 0.56 & 0.68 & 0.61 & 0.67 & 0.84 & 0.71 & 0.36 & 0.67 & 0.44 & 0.57 & 0.24 \\
\hline trellis 70 & 0.61 & 0.22 & 0.39 & 0.35 & 0.46 & 0.29 & 0.50 & 0.60 & 0.38 & 0.21 & 0.72 & 0.32 & 0.62 & 0.39 & 0.63 \\
\hline surfing & 0.88 & 0.78 & 0.84 & 0.79 & 0.82 & 0.50 & 0.87 & 0.84 & 0.85 & 0.60 & 0.79 & 0.40 & 0.73 & 0.82 & 0.48 \\
\hline sphere & 0.70 & 0.42 & 0.54 & 0.36 & 0.60 & 0.08 & 0.68 & 0.56 & 0.18 & 0.49 & 0.68 & 0.06 & 0.11 & 0.64 & 0.66 \\
\hline singer & 0.78 & 0.45 & 0.48 & 0.41 & 0.18 & 0.26 & 0.46 & 0.86 & 0.70 & 0.40 & 0.47 & 0.47 & 0.73 & 0.46 & 0.29 \\
\hline girl & 0.73 & 0.32 & 0.68 & 0.45 & 0.53 & 0.60 & 0.41 & 0.71 & 0.68 & 0.59 & 0.35 & 0.38 & 0.73 & 0.71 & 0.25 \\
\hline football & 0.65 & 0.02 & 0.64 & 0.52 & 0.23 & 0.59 & 0.60 & 0.66 & 0.45 & 0.60 & 0.57 & 0.68 & 0.58 & 0.56 & 0.35 \\
\hline faceocc 2 & 0.73 & 0.54 & 0.79 & 0.72 & 0.59 & 0.38 & 0.77 & 0.74 & 0.67 & 0.57 & 0.77 & 0.78 & 0.77 & 0.77 & 0.46 \\
\hline david & 0.60 & 0.41 & 0.36 & 0.42 & 0.43 & 0.23 & 0.38 & 0.53 & 0.50 & 0.60 & 0.50 & 0.57 & 0.45 & 0.64 & 0.58 \\
\hline carchase & 0.87 & 0.29 & 0.44 & 0.53 & 0.82 & 0.60 & 0.85 & 0.58 & 0.59 & 0.80 & 0.84 & 0.40 & 0.79 & 0.72 & 0.31 \\
\hline car4 & 0.89 & 0.24 & 0.74 & 0.27 & 0.22 & 0.23 & 0.49 & 0.80 & 0.62 & 0.57 & 0.47 & 0.23 & 0.87 & 0.49 & 0.15 \\
\hline car11 & 0.77 & 0.00 & 0.51 & 0.22 & 0.55 & 0.10 & 0.83 & 0.80 & 0.52 & 0.28 & 0.80 & 0.52 & 0.79 & 0.82 & 0.43 \\
\hline biker & 0.68 & 0.45 & 0.31 & 0.43 & 0.44 & 0.27 & 0.38 & 0.44 & 0.39 & 0.30 & 0.45 & 0.27 & 0.39 & 0.37 & 0.42 \\
\hline bicycle & 0.59 & 0.33 & 0.32 & 0.54 & 0.31 & 0.11 & 0.40 & 0.64 & 0.29 & 0.39 & 0.25 & 0.25 & 0.54 & 0.28 & 0.35 \\
\hline human & 0.78 & 0.33 & 0.66 & 0.48 & 0.54 & 0.47 & 0.53 & 0.65 & 0.73 & 0.08 & 0.53 & 0.31 & 0.74 & 0.49 & 0.23 \\
\hline osow & 0.92 & 0.56 & 0.83 & 0.56 & 0.71 & 0.77 & 0.81 & 0.89 & 0.91 & 0.65 & 0.81 & 0.82 & 0.90 & 0.80 & 0.54 \\
\hline
\end{tabular}

and the overlapping rate. The center location error is the Euclidean distance between the center of the tracking result and the ground truth for each frame. The overlapping rate is based on the PASCAL challenge object detection score [8]. Given the tracked bounding box $R O I_{T}$ and the ground truth bounding box $R O I_{G T}$, the overlap score is computed by score $=\frac{\operatorname{area}\left(R O I_{T} \cap R O I_{G T}\right)}{\operatorname{area}\left(R O I_{T} \cup R O I_{G T}\right)}$. To rank the tracking performance, we compute the average center location error and the average overlap score across all frames of each image sequence as done in $[23,22,34,37,15,13]$. These results on the 20 image sequences are summarized in Table 1. Overall, the proposed SST algorithm performs favorably against the other state-of-the-art algorithms on all tested sequences. Due to space limitation, the average center location errors and more experimental results as well as videos are available in the supplementary material.

The comparison results on benchmark [30] are shown in Figure 6. The results show that our SST tracker achieves favorable performance than other related sparse trackers [23, 22, 34, 37, 4, 15]. Compared with other state-of-the-art methods, our SST achieves the second best overall performance. We note that the SST algorithm performs well for the videos with background clutter, illumination variations, low resolution, and occlusions attributes based on the precision metric as shown in Figure 7. Similarly, the SST algorithm achieves favorable results for the videos with the above attributes using the success rate metric as shown in Figure 8.

\subsection{Qualitative Evaluation}

Figure 5 shows some tracking results of 15 trackers on 20 image sequences. The tracking results are discussed below based on the main challenging factors in each video.

Occlusion. In the olsr sequence, all the other trackers lose track of the target person at frame 200 as she is partially occluded by a man. As the other trackers lock onto the man, the errors increase for the rest of the sequence, as shown in Figure 5. In the $t u d$ sequence, where the target vehicle is occluded by crossing pedestrians. The MIL, OAB, CST, LST,
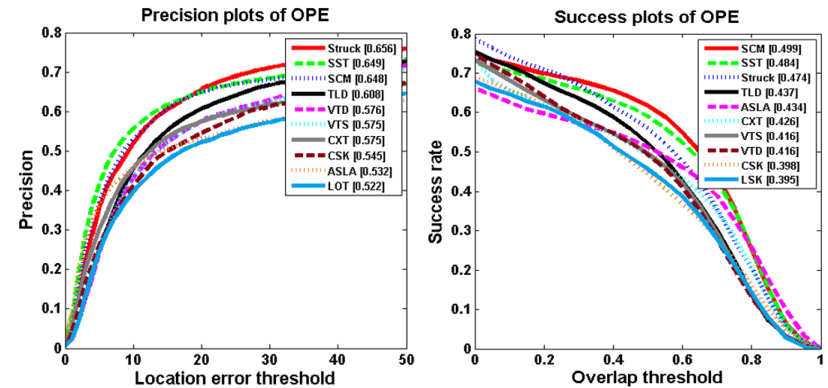

(a) global sparse appearance model (b) global sparse appearance model

Figure 6. Precision and success plots of overall performance comparison for the 51 videos in the benchmark [30] (best-viewed on high-resolution display). The mean precision scores for each tracker are reported in the legends. Note that, our SST tracker improves the baseline sparse trackers and achieves the best performance. Moreover, our approach achieves the second best and performs favorably to other state-of-the-art tracking methods.

Struck, and RTCT methods drift away from the target objec$\mathrm{t}$ when occlusion occurs. On the other hand, the $\ell_{1}$, DFT, Frag, and the proposed SST methods perform well in this sequence. In the other sequences with occlusion, e.g., osow, the proposed SST performs at least the second best.

Scale change. The human and sphere videos contain significant scale change. In the human sequence, a person walks away from the camera and is occluded by the pole for a short duration. The TLD method loses track of the target from the start, the RTCT drifts at frame 1674, and the DFT and LGT trackers start to drift off the target at frame 1600 and finally loses track of the target. All the other methods successfully track the target but the SST, $\ell_{1} \mathrm{~T}$, LST, and IVT methods achieve higher overlap scores. In the sphere sequence, the scale of the sphere changes significantly. While most trackers fail to track the ball, the SST, CST, and Struck methods can track the target throughout this sequence.

Abrupt motion. In the football and tunnel sequences, the target objects undergo abrupt motion in cluttered backgrounds. In the football sequence, several objects similar to 


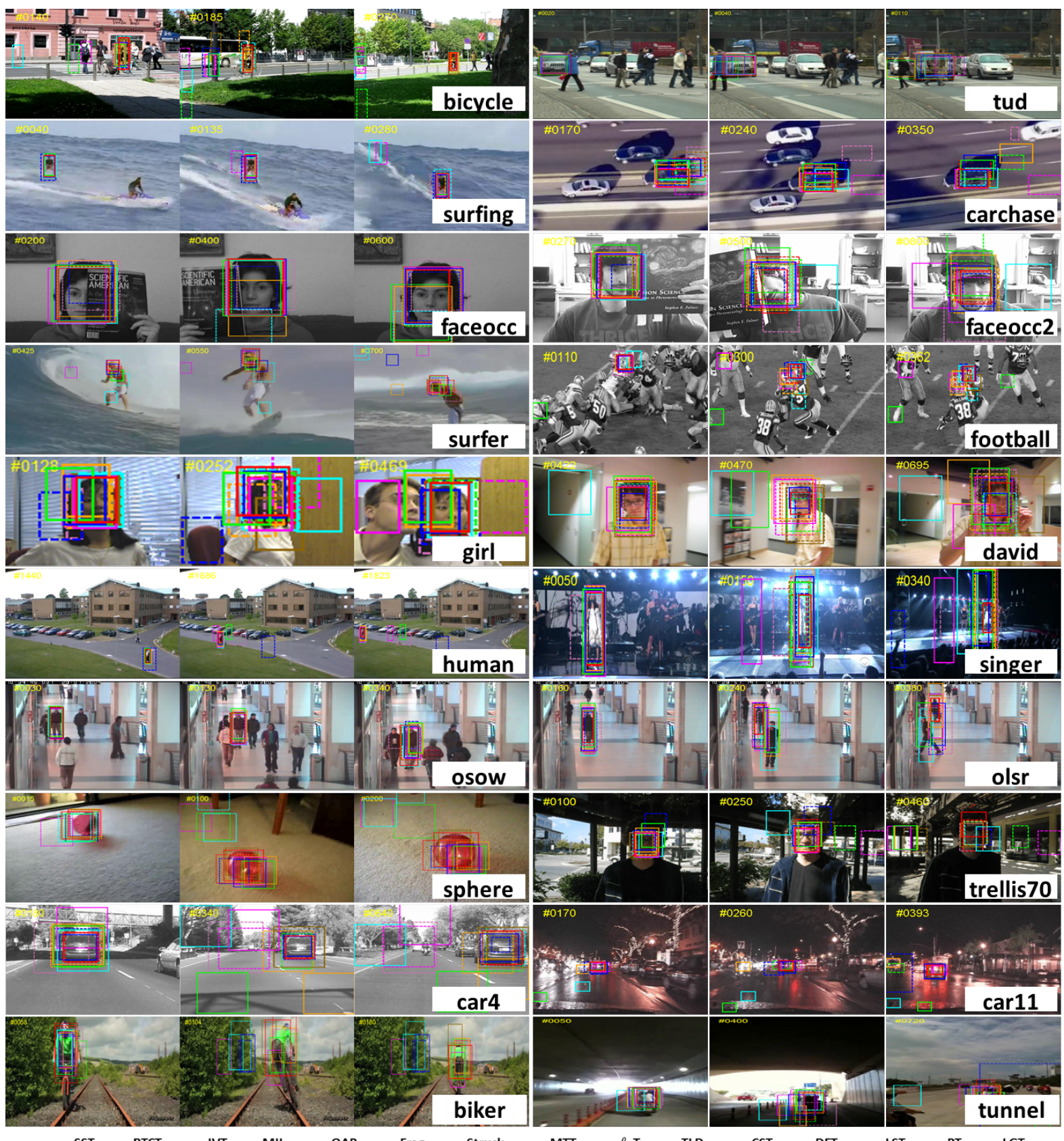

Figure 5. Tracking results of 15 trackers (denoted in different colors and lines) on 20 image sequences. Frame indexes are shown in the top left of each figure in yellow color. See text for details. Results are best viewed on high-resolution displays.

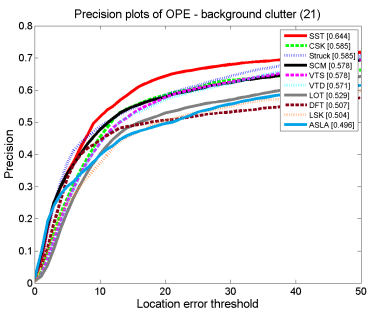

(a) background clutter

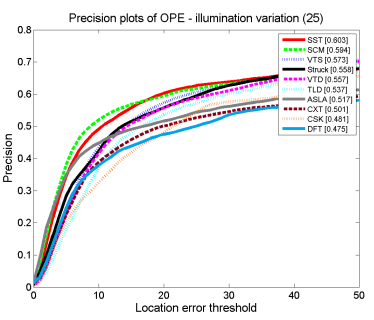

(b) illumination variations

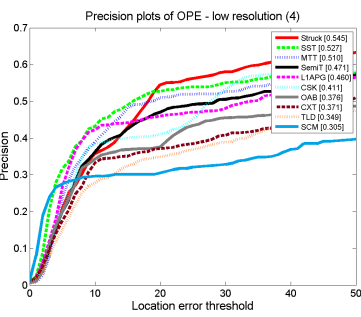

(c) low resolution

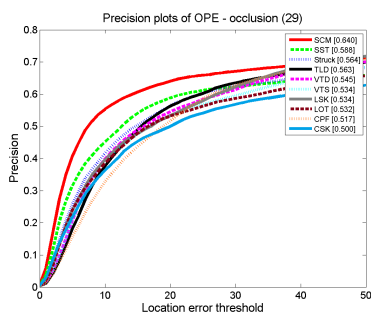

(d) occlusions

Figure 7. The plots of OPE with attributes based on the precision metric. 


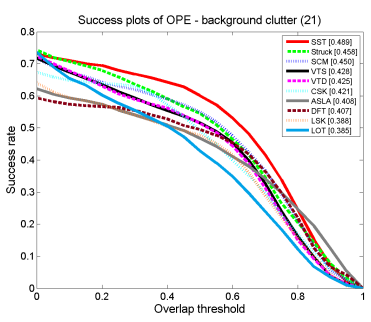

(a) background clutter

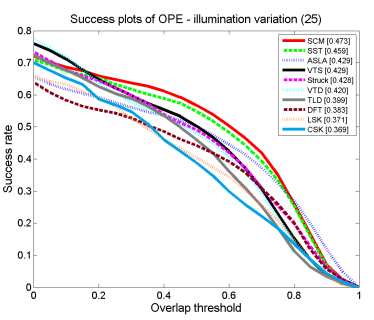

(b) illumination variations

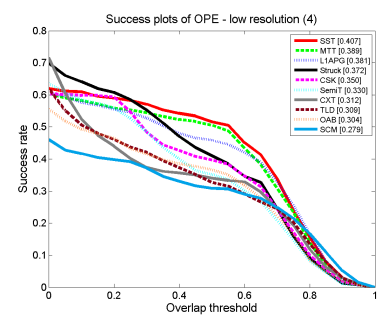

(c) low resolution

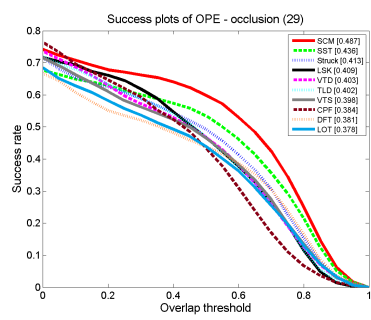

(d) occlusions

Figure 8 . The plots of OPE with attributes using the success rate metric.

the target appear in the scenes. The OAB, $\ell_{1} \mathrm{~T}$ and LGT algorithms drift at frame 100, 246 and 276, respectively. Most methods drift from the intended target to other objects when they appear in close proximity (e.g., frame 362 ). The proposed SST, TLD, DFT, and MTT methods successfully track the target for the entire sequence.

Illumination and scale changes. The car4, carll, and singer image sequences contain large illumination and scale changes. For the car4 sequence, the OAB, Frag, and LGT methods start to drift from the target at frame 185 when the vehicle goes through the overpass. The MIL, DFT, and RTC$\mathrm{T}$ algorithms drift away from the target object at frame 210 . The $\ell_{1}$ T, PT, CST, Struck, and TLD approaches are able to track the target well in most frames. On the other hand, the target object is successfully tracked by the LST, IVT, MTT, and SST algorithms throughout the entire sequence despite large illumination changes.

Illumination change and pose variation. The david and trellis70 sequences contain illumination change and pose variation. In the david sequence, the Frag, RTCT, and OAB methods fail around frames 330,445 , and 550, respectively. The MIL and $\ell_{1}$ trackers adequately track the face, but experience some drift. The SST, TLD, PT, and LGT methods track the moving face accurately throughout this sequence. In the trellis 70 sequence, the Frag and RTCT methods drift away from the target when significant lighting change occurs. Due to the combination of lighting and head pose changes, the IVT, Frag, TLD, PT, and DFT methods fail to track the target, especially toward the latter part of this sequence. In contrast, the SST, LST, MTT, CST, and LGT methods successfully track the target for whole video.

Occlusion and pose variation. The faceocc 2 and bicycle sequences contain significant occlusion and pose variation. Tracking results on the faceocc 2 sequence show that most trackers drift away from the target when it is heavily occluded. As the proposed SST algorithm exploits structural sparse appearance model to account for occlusion, it performs well in this sequence. The bicycle sequence contains scenes with heavy occlusion and pose variation. The SST, LST, Struck, and MTT methods are capable of tracking the target for the entire sequence. Other trackers experience drift at differen$\mathrm{t}$ time instances (Frag at frame 40, $\ell_{1} \mathrm{~T}$ at frame 132, DFT at frame 135, MIL and CST at frame 144, other trackers at frame 177 due to the occlusion).

Abrupt motion, pose variation, and occlusion. The girl and carchase sequences contain objects with abrupt motion, pose variation, and occlusion. In the girl sequence, the SST, LST, MTT, and PT methods are capable of tracking the target for the entire sequence whereas other methods gradually drift away. On the carchase sequence, the DFT method starts to drift at frame 139. The IVT, LGT, and MTT algorithms drift at frame 169 when occlusion occurs. The OAB, CST, TLD, Struck, and SST methods perform well throughout this sequence. Most of the other trackers have drift problem due to the abrupt motion and pose variation.

\section{Conclusion}

In this paper, we propose a novel structural sparse appearance model for object tracking within the particle filter framework, where the representations of target candidates and their image patches, regularized by a sparsity-inducing $\ell_{2,1}$ mixed norm, are learned jointly by use of an efficient Accelerated Proximal Gradient (APG) method. We show that this model exploits the intrinsic relationship among not only target candidates, but also the corresponding local patches to learn their sparse representations jointly. In addition, the proposed model preserves the spatial layout structure among the local patches inside each target candidate. The proposed algorithm is general and accommodates most existing sparse trackers. Experimental results with evaluations against several state-of-the-art methods on challenging image sequences demonstrate the effectiveness and robustness of the proposed SST tracking algorithm. In the future, we will extend the proposed structural sparse appearance model for object tracking by considering noises, random part sampling, etc.

\section{Acknowledgment}

This study is supported by the research grant for the $\mathrm{Hu}$ man Sixth Sense Programme at the Advanced Digital Sciences Center from Singapore's Agency for Science, Technology and Research (A*STAR). C. Xu is supported by 973 Program Project No. 2012CB316304 and NSFC 61225009, 61432019, 61303173, U1435211, 173211KYSB20130018. M.-H. Yang is supported in part by NSF CAREER Grant \#1149783 and NSF IIS Grant \#1152576. 


\section{References}

[1] A. Adam, E. Rivlin, and I. Shimshoni. Robust fragmentsbased tracking using the integral histogram. In $C V P R$, pages 798-805, 2006. 1, 5

[2] S. Avidan. Ensemble tracking. In CVPR, pages 494-501, 2005. 1

[3] B. Babenko, M.-H. Yang, and S. Belongie. Visual tracking with online multiple instance learning. In $C V P R, 2009.1,5$

[4] C. Bao, Y. Wu, H. Ling, and H. Ji. Real time robust $l_{1}$ tracker using accelerated proximal gradient approach. In $C V P R$, 2012. 1, 2, 3, 6

[5] M. J. Black and A. D. Jepson. Eigentracking: Robust matching and tracking of articulated objects using a view-based representation. IJCV, pages 63-84, 1998. 1

[6] R. T. Collins and Y. Liu. On-line selection of discriminative tracking features. In ICCV, pages 346-352, 2003. 1

[7] D. Comaniciu, V. Ramesh, and P. Meer. Kernel-Based Object Tracking. TPAMI, 25(5):564-575, Apr. 2003. 1

[8] M. Everingham, L. Gool, C. Williams, J. Winn, and A. Zisserman. The pascal visual object class (voc) challenge. IJCV, 88(2):303-338, 2010. 6

[9] H. Grabner, M. Grabner, and H. Bischof. Real-Time Tracking via On-line Boosting. In $B M V C$, 2006. 1, 5

[10] H. Grabner, C. Leistner, and H. Bischof. Semi-supervised online boosting for robust tracking. In $E C C V, 2008.1$

[11] S. Hare, A. Saffari, and P. Torr. Struck: Structured output tracking with kernels. In ICCV, 2011. 5

[12] J. Henriques, R. Caseiro, P. Martins, and J. Batista. Exploiting the circulant structure of tracking-by-detection with kernels. In $E C C V, 2012.5$

[13] Z. Hong, X. Mei, D. Prokhorov, and D. Tao. Tracking via robust multi-task multi-view joint sparse representation. In ICCV, 2013. 1, 2, 3, 5, 6

[14] A. Jepson, D. Fleet, and T. El-Maraghi. Robust on-line appearance models for visual tracking. TPAMI, 25(10):12961311, 2003. 1

[15] X. Jia, H. Lu, and M.-H. Yang. Visual tracking via adaptive structural local sparse appearance model. In CVPR, 2012. 1, 2, 3, 4, 5, 6

[16] Z. Kalal, J. Matas, and K. Mikolajczyk. P-N Learning: Bootstrapping Binary Classifiers by Structural Constraints. In CVPR, 2010. 1, 5

[17] M. Kristan, L. Cehovin, and et al. The visual object tracking vot 2013 challenge results. In ICCV2013 Workshops, Workshop on Visual Object Tracking Challenge, 2013. 1

[18] J. Kwon and K. M. Lee. Visual tracking decomposition. In CVPR, 2010. 1

[19] H. Li, C. Shen, and Q. Shi. Real-time visual tracking with compressed sensing. In $C V P R, 2011.1,2,3,5$

[20] B. Liu, J. Huang, L. Yang, and C. Kulikowski. Robust visual tracking with local sparse appearance model and k-selection. In $C V P R, 2011.1,2,3,4,5$

[21] B. Liu, L. Yang, J. Huang, P. Meer, L. Gong, and C. Kulikowski. Robust and fast collaborative tracking with two stage sparse optimization. In ECCV, 2010. 1, 2, 3, 5

[22] X. Mei and H. Ling. Robust Visual Tracking and Vehicle Classification via Sparse Representation. TPAMI, 33(11):22592272, 2011. 1, 2, 3, 4, 5, 6
[23] X. Mei, H. Ling, Y. Wu, E. Blasch, and L. Bai. Minimum error bounded efficient 11 tracker with occlusion detection. In CVPR, 2011. 1, 2, 3, 5, 6

[24] Y. Pang and H. Ling. Finding the best from the second best$\mathrm{s}$ - inhibiting subjective bias in evaluation of visual tracking algorithms. In ICCV, 2013. 1

[25] D. Ross, J. Lim, R.-S. Lin, and M.-H. Yang. Incremental Learning for Robust Visual Tracking. IJCV, 77(1):125-141, 2008. 1, 5

[26] S. Salti, A. Cavallaro, and L. D. Stefano. Adaptive appearance modeling for video tracking: Survey and evaluation. TIP, 21(10):4334-4348, 2012. 1

[27] L. Sevilla-Lara and E. Learned-Miller. Distribution fields for tracking. In CVPR, pages 1910-1917, 2012. 5

[28] L. Čehovin, M. Kristan, and A. Leonardis. Robust visual tracking using an adaptive coupled-layer visual model. TPAMI, 35(4):941-953, 2013. 5

[29] D. Wang, H. Lu, and M. Yang. Online Object Tracking with Sparse Prototypes. IEEE Transaction on Image Processing, 22(1):314-325, 2013. 1

[30] Y. Wu, J. Lim, and M.-H. Yang. Online object tracking: A benchmark. In $C V P R, 2013.1,6$

[31] M. Yang, Y. Wu, and G. Hua. Context-aware visual tracking. TPAMI, 31(7):1195-1209, 2009. 1

[32] R. Yao, Q. Shi, C. Shen, Y. Zhang, and A. van den Hengel. Part-based visual tracking with online latent structural learning. In $C V P R, 2013.5$

[33] A. Yilmaz, O. Javed, and M. Shah. Object tracking: A survey. ACM Comput. Surv., 38(4):13, Dec. 2006. 1

[34] K. Zhang, L. Zhang, and M.-H. Yang. Real-time compressive tracking. In $E C C V, 2012$. 1, 2, 3, 5, 6

[35] T. Zhang, B. Ghanem, and N. Ahuja. Robust multi-object tracking via cross-domain contextual information for sport$\mathrm{s}$ video analysis. In International Conference on Acoustics, Speech and Signal Processing, 2012. 1

[36] T. Zhang, B. Ghanem, S. Liu, and N. Ahuja. Low-rank sparse learning for robust visual tracking. In ECCV, 2012. 1, 2, 3, 5

[37] T. Zhang, B. Ghanem, S. Liu, and N. Ahuja. Robust visual tracking via multi-task sparse learning. In $C V P R, 2012$. 1, 2, $3,5,6$

[38] T. Zhang, B. Ghanem, S. Liu, and N. Ahuja. Robust visual tracking via structured multi-task sparse learning. International Journal of Computer Vision, 101(2):367-383, 2013. 3

[39] T. Zhang, C. Jia, C. Xu, Y. Ma, and N. Ahuja. Partial occlusion handling for visual tracking via robust part matching. In CVPR, 2014. 1

[40] T. Zhang, S. Liu, N. Ahuja, M.-H. Yang, and B. Ghanem. Robust Visual Tracking via Consistent Low-Rank Sparse Learning. International Journal of Computer Vision, 111(2):171190, 2015. 3

[41] W. Zhong, H. Lu, and M. Yang. Robust Object Tracking via Sparse Collaborative Appearance Model. IEEE Transaction on Image Processing, 23(5):2356-2368, 2014. 1

[42] B. Zhuang, H. Lu, Z. Xiao, and D. Wang. Visual Tracking via Discriminative Sparse Similarity Map. IEEE Transaction on Image Processing, 23(4):1872-1881, 2014. 1 